\title{
Quinine doped hybrid sol-gel coatings for wave guiding and optical applications
}

\author{
E. Enríquez $\cdot$ M. A. Garcia $\cdot$ N. Carmona \\ J. F. Fernández $\cdot$ M. A. de la Rubia
}

\begin{abstract}
Pure and quinine doped silica coatings have been prepared over sodalime glasses. The coatings were consolidated at low temperature (range $60-180{ }^{\circ} \mathrm{C}$ ) preserving optical activity of quinine molecule. We designed a device to test the guiding properties of the coatings. We confirmed with this device that light injected in pure silica coatings is guided over distances of meters while quinine presence induces isotropic photoluminescence. With the combined use of both type of coatings, it is possible to design light guiding devices and illuminate regions in glass elements without electronic circuits.
\end{abstract}

Keywords Sol-gel coatings - Photoluminescence . Waveguides · Silica $\cdot$ Quinine

\section{Introduction}

Nowadays optical fibers are the preferred technology for information transmission due the high speed, wide bandwidth and low power consumption of optical signals $[1,2]$.
Moreover, optical beams traveling along waveguides are insensitive to outward disturbances as electric fields, water vapor, oxygen or particles, rending optical transmission as a key element of information society. Light transmission is also an interesting solution where electrical lines are not suitable because of security problems. There are well established methods to fabricate waveguides based on ion exchange, films deposition, photolithography that are appropriated for high added value products [3-8]. However, further development of this technology relays on the ability to create waveguides over large surfaces at low cost.

Sol-gel is an outstanding process to prepare coatings at reasonable cost [9-12]. Depositing a glass coating with the proper refraction index over a glassy substrate it is possible to obtain an interface with the required conditions to exhibit total internal reflection, confining the light inside the glassy substrate or in the coating [13-15]. Moreover, sol-gel coatings fulfill most of the requirements for optical wave guiding as they are good adhesion, a proper thickness and roughness control, and the possibility to encapsulate dispersed nanostructures in the coating in order to tune its optical properties [4, 16-19].

In this work we address the fabrication of waveguides by deposition of silica coatings onto sodalime glasses ( $14 \%$ sodium carbonate, $9 \%$ calcium oxide, $4 \%$ magnesium oxide, $73 \%$ silica and $0,15 \%$ alumina, and a refractive index of 1,520 ) using a sol-gel route. A systematic study of the relationship between preparation conditions and optical performance of the system is also presented. A fluorescent molecule, as quinine, is introduced in the silica matrix in order to induce photoluminescence in the coating. We present a home-made device to test the efficiency of the coating as waveguide. With these results, we propose a design to explode these materials in the controlled illumination of glass elements. 


\section{Experimental}

Sol-gel silica coatings were prepared using tetraethyl orthosilicate (TEOS, Sigma Aldrich) as silica precursor, ethanol absolute $99.9 \%$ (MERCK) as dissolvent and distilled water to promote the hydrolysis [20]. The mol ratio TEOS: EtOH: $\mathrm{H}_{2} \mathrm{O}$ was 1:4:3. Quinine molecule (Q, Sigma Aldrich) was added in some sols to a final concentration ranging from 1 to $15 \mathrm{wt}$. \% to silica. In this case, sulfuric acid was used as the catalyst, and it plays double role because it not only works as catalyst but also improves the luminescence of the molecule as substituting groups are key in the luminescence, especially groups that lose electrons because they increase the probability of transition from the excited singlet to fundamental state, improving the luminescence. In general, an increase of rigidity in the structure of molecules results in an increase on probability of fluorescence because it minimizes vibrations and crossing systems [21, 22]. Final solutions were stirred $2 \mathrm{~h}$ to allow hydrolysis and polycondensation. Substrates were sodalime microcopy slides $\left(\mathrm{SiO}_{2} 73 \%, \mathrm{Na}_{2} \mathrm{O} 14 \%, \mathrm{CaO}\right.$ $7 \%, \mathrm{MgO} 4 \%, \mathrm{Al}_{2} \mathrm{O}_{3} 2 \%$, refractive index 1.52) with $20 \mathrm{~mm} \times 70 \mathrm{~mm}$ dimensions. Prior to coating deposition, glass substrates were cleaned by immersion in ethanol and acetone baths successively and dried at room temperature. Coatings were applied by dip-coating with an extraction speed of $2.5 \mathrm{~mm} / \mathrm{s}$, which allows to tailor the coating thickness [23]. Densification treatments in air were carried out at $60,100,150$ and $180^{\circ} \mathrm{C}$ for times ranging between 5 and $48 \mathrm{~h}$. Multilayers were also prepared by deposition of additional layers after the thermal treatments of the previous layers. Coatings roughness was modified etching of the substrate with $\mathrm{HF}$ (10 and $40 \%$ vol) prior to coating deposition. In order to study the effect of the preparation conditions on the guiding properties, sets of samples varying different parameters as annealing temperature, number of layers, quinine concentration and HF etching conditions were prepared and characterized.

Thermal Gravimetry Analysis (TGA) of the sol dried was performed using Netzsch STA 409/C, in the range $30-1,000{ }^{\circ} \mathrm{C}$. Structural characterization was carried out by means of Hitachi TM-1000 Scanning Electron Microscopy (SEM) and atomic force microscopy (AFM) images were obtained with a NT-MDT Solver SPM instrument with antimony doped single crystal silicon tips for contact mode with $\mathrm{Au}$ reflective side and resonant frequency $4-17 \mathrm{kHz}$. The coatings thicknesses were determined by optical interference as described in [24] using a Perkin Elmer Lambda 950 spectrophotometer with integrating sphere. Refractive indexes were obtained using an ellipsometer M-2000U (J.A. Woollan Co.) The wave guiding properties were measured with a home-made device described in Sect. 3 .

\section{Results and discussion}

Figure 1 presents a DTA-TG of a sol dried xerogel of silica (Fig. 1a), commercial quinine anhydrous powders (Fig. 1b) and silica-quinine $12 \%$ wt (Fig. 1c). Figure 1a shows that silica begins to lose weight gradually from $50^{\circ} \mathrm{C}$ which is related to the evaporation of organic matter from the initial precursors (dehydration and alcohol evaporation). DTA analysis shows an endothermic peak at $140{ }^{\circ} \mathrm{C}$ due to the removal of silanol $\mathrm{OH}$ groups, and the hydration water molecules. There is a progressive weight loss up to $600{ }^{\circ} \mathrm{C}$ when the film is fully densified. The onset of the polycondensation of silica ( $\mathrm{Si}-\mathrm{O}-\mathrm{Si})$ takes place from $\sim 320^{\circ} \mathrm{C}$ reaching the end of the process at $370^{\circ} \mathrm{C}[25$, 26]. The onset of the quinine degradation takes places from $243{ }^{\circ} \mathrm{C}$ (exothermic peak) as it can be seen in Fig. 1b, due to the decomposition of the quinoline moiety (heterocyclic aromatic compound belonging to quinine molecule), which is accompanied by a weight loss of $14 \mathrm{wt} \%$, in concordance with literature [27, 28]. The greatest weight loss (above $70 \%$ ) occurs from $373{ }^{\circ} \mathrm{C}$, reaching the end of the decomposition at $497^{\circ} \mathrm{C}$. According to Fig. 1c, silica matrix protects the quinine molecule, delaying its degradation since the exothermic peak at $497^{\circ} \mathrm{C}$ is displaced to $541{ }^{\circ} \mathrm{C}$. The degradation of the quinoline at $270{ }^{\circ} \mathrm{C}$ is not possible to identify in Fig. 1c due to the protection by the silica matrix, which reduces the degradation of this group. Therefore, coatings containing this molecule cannot be consolidated above this temperature $\left(180^{\circ} \mathrm{C}\right)$. Thus, our films are not pure silica but the content about $10 \%$ of organic matter, mainly carbon compounds [29].

Refraction indices were measured by ellipsometry. For the samples annealed at $60^{\circ} \mathrm{C}$ for $48 \mathrm{~h}$ we found $\mathrm{n}=1.451 \pm 0.004$ without a clear dependence on the quinine concentration in the range $0-15 \%$ wt. Using this value of refraction index, the coatings thicknesses were determined from the interferences in the optical reflectance spectra as described in [24]. The thickness of the individual layers resulted of the order of $500 \mathrm{~nm}$. As it can be seen in Fig. 2, for the pure silica multilayers there is a perfect linear relationship between the multilayer thickness and the number layers, (being the slope the thickness of the single layer). For the coatings with $12 \%$ of quinine the behavior is about the same although there some deviation from the linear behavior. This behavior could be related to sol ageing or local inhomogeneities leading to variations of viscosity and consequently on the film thickness [23]. Note that multilayers are annealed after the deposition of each layer, so we must keep the sol several hours between the depositions of each layer.

For coatings sintered at higher temperatures, the linear relationship between thickness and the number of layers, remains, but the multilayer thickness decreases due to the 
(a)

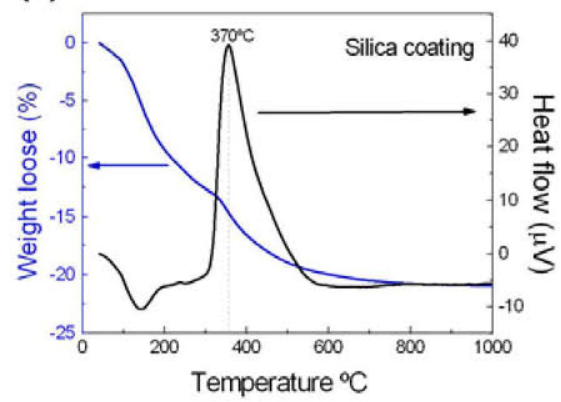

(b)

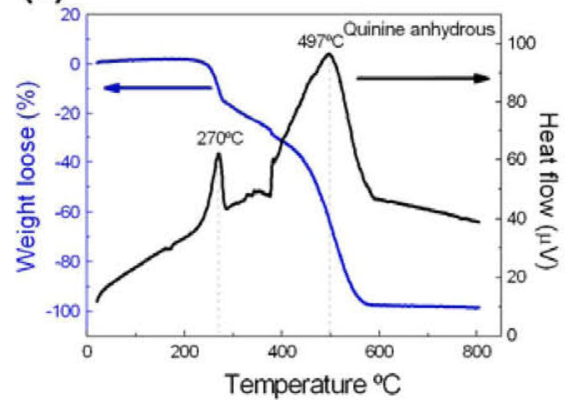

(c)

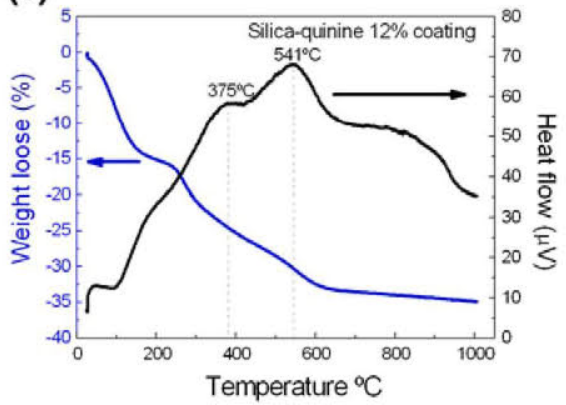

Fig. 1 DTA-TG of a a pure silica coating, b commercial quinine anhydrous powder and $\mathbf{c}$ silica-quinine $12 \%$ wt coating (under air at a heating rate of $3{ }^{\circ} \mathrm{C} / \mathrm{min}$ )

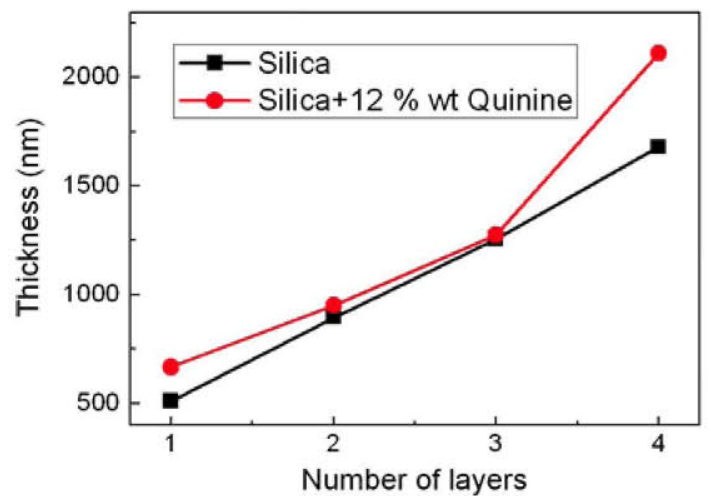

Fig. 2 Thickness of pure silica and quinine doped (12\%) coatings densified at $60^{\circ} \mathrm{C}$ for $48 \mathrm{~h}$

better densification of the coating [30, 31]. In particular, the slope of thickness vs number of layers for coatings sintered at 60 and $180{ }^{\circ} \mathrm{C}$ is $392 \mathrm{~nm} /$ layer and $345 \mathrm{~nm} /$ layer, respectively.

With the deposition of multilayer it is possible to increase the coating thickness up to $3 \mu$. Actually above $2 \mu$ the thickness was not reproducible and the layers loose adherence leading to inhomogeneous, rough and mechanically poor coatings.

The effect of the substrate etching on the coating morphology was studied by SEM and AFM at different length scales. SEM micrographs presented in Fig. 3 confirms that coatings deposited onto smooth substrates (Fig. 3a, c) reproduce the glass topography resulting continuous and flat surfaces. On the contrary, the coatings deposited onto etched substrates ( $\mathrm{HF}$ 40\% vol, $20 \mathrm{~s}$ ) exhibit significant roughness and the film is not continuous with some regions were the substrate is uncovered as illustrated in figures (Fig. 3b, d). At this length scale the roughness induced by the substrate etching is independent of the quinine content.

Figures 4 and 5 present AFM images of the substrates etched ( $20 \mathrm{~s}$ and $2 \mathrm{~min}, 40 \% \mathrm{HF}$ ) and for silica coatings deposited onto these substrates. Upon $20 \mathrm{~s}$ chemical etching, the substrate presents pores of the order of 1 micron that cover about $36 \%$ of the surface. The coatings deposited on these etched substrates exhibits pores typically $2 \mu$ wide and $40 \mathrm{~nm}$ deep, covering at about $6 \%$ of the surface. If the etching time is increased up to $2 \mathrm{~min}$, the substrate exhibits pores of the order of $2-4 \mu$ covering $60 \%$ of the surface while for the corresponding coating the pores are typically $6-8 \mu$ size covering roughly $40 \%$ of the surface.

As expected, we observed that the pores density and size increase with the etching time. Surface defects are preferential etching points leading to the formation of pores during the first stages of the attack. As the etching time increases, these pores percolate increasing their effective size. The coatings exhibit a smaller concentration of pores than the substrate but they result larger. It turns out that for the regions of the substrate with a large density of pores, the coatings exhibit a large pore that corresponds to the percolation of the pores in this region of the substrate. On the contrary, for the areas of the substrate with a reduced density of pores the coating fill these pores leading to a flat surface.

The morphology of the coatings with quinine deposited on etched substrate is identical to that of pure silica ones. Therefore, we conclude that the presence of quinine does not alter significantly the morphology of the coatings. Variations in the quinine concentration does not alter viscosity enough to promote variations in the surface morphology.

Quinine is known to exhibit intense fluorescence with emission in the blue region of the spectrum upon excitation with UV light [20, 32-35]. Figure 6 presents the photoluminescence spectrum of silica with different quinine concentrations (1-15\%wt). It can be observed that the PL intensity increases with the quinine concentration reaching the highest intensity for sample with $12 \%$ wt and decreasing for higher concentrations. This decrease is due to a quenching for concentration (fluorescence inhibition) which 
Fig. 3 SEM images of (a and b) pure silica and (c and d) quinine doped $(12 \%)$ coatings deposited on (a and c) smooth substrates and (b and d) chemically etched ( $\mathrm{HF} 40 \%$ vol, 20 s.) substrates
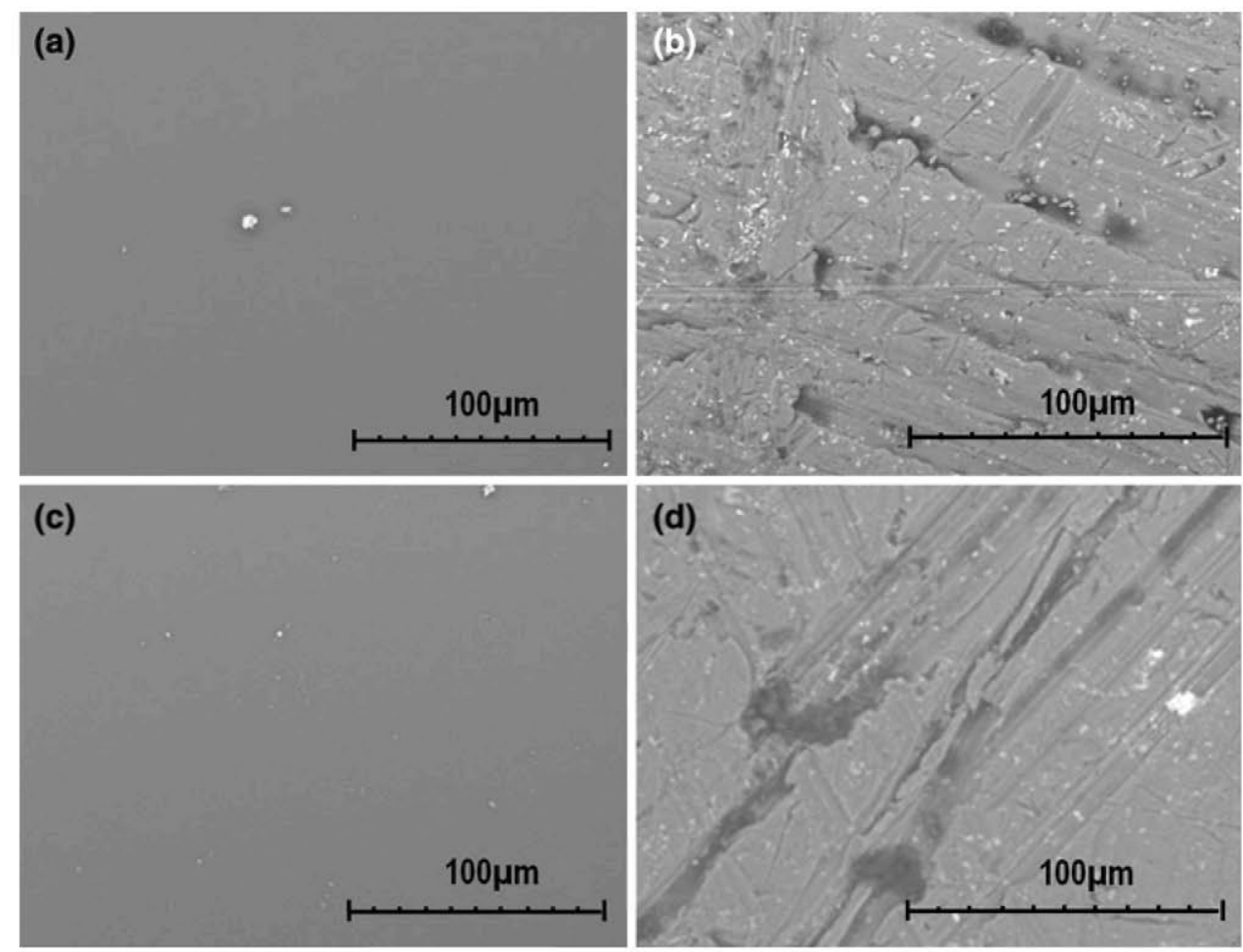

appears when concentration reaches a maximum from which there is an significant self-absorption of fluorescent radiation by the solution [36]. In the inset of Fig. 6a it can be seen an enlargement of emission curves for samples with lower luminescent intensity. We find the maximum of the emission spectrum at $433 \mathrm{~nm}$ while the maximum of the excitation spectrum is located at $355 \mathrm{~nm}$. The shoulder in the excitation band, around $320 \mathrm{~nm}$, is due to transitions to higher energies, to second excited states, which do not reflects in the emission spectrum because of a rapid relaxation of these transitions to first excited states [37, 38]. The position of these bands is in agreement with previous works $[20,32]$ that showed displacements of the optical bands for quinine depending on the features of the medium.

In order to asses the wave guiding behavior of the material, we designed the device presented in Fig. 7. The coating is allocated into a cavity with holes to illuminate the sample in a controlled way. Light guided inside the material travels out of the cavity and may escape from the device through the glass edges. Measuring the light leaving the sample as a function of the distance to the illumination region with a photodiode we may estimate the performance of the system for wave guiding. To this purpose we assume two hypotheses: (a) the light leaving the material at a certain distance of the illumination point is proportional of the light intensity reaching this point; (b) light decay due to edges is significantly smaller than that produced in the rest of the material. We used this device to test guiding properties of two kinds of materials: Pure silica coatings and silica doped with quinine ones. The device has been designed with the following components: a photodiode of silicon with wavelength range of (40-1100) $\mathrm{nm}$, an active area of $9.7 \mathrm{~mm} \times 9.7 \mathrm{~mm}$, rise time of $40 \mathrm{~ns}$ and bandwidth of $8 \mathrm{MHz}$; two light sources, depending on the coating composition, for quinine containing coating it is used LEDs with maximum emission in $365 \mathrm{~nm}$ and a wide band of FWHM of $10 \mathrm{~nm}$ with $180 \mathrm{~mW}$ of power, and for silica coating, it is used LEDs of $370 \mathrm{~nm}$, near to visible, with FWHM of $10 \mathrm{~nm}$, and $110 \mathrm{~mW}$ of power.

For silica coatings we injected horizontally the light to be guided into the material (Fig. 7a). The sample is illuminated parallel to the substrate plane in order to maximize the number of photons reaching the film with a high angle with respect to the normal, i.e., with incidence angle over the total reflection one, to promote light guiding inside the glass.

For the quinine containing coatings, we used the device of Fig. 7b illuminating vertically the coating with UV light to promote the photoluminescence light emission. In this case, as photoluminescence emission is about isotropic it is 

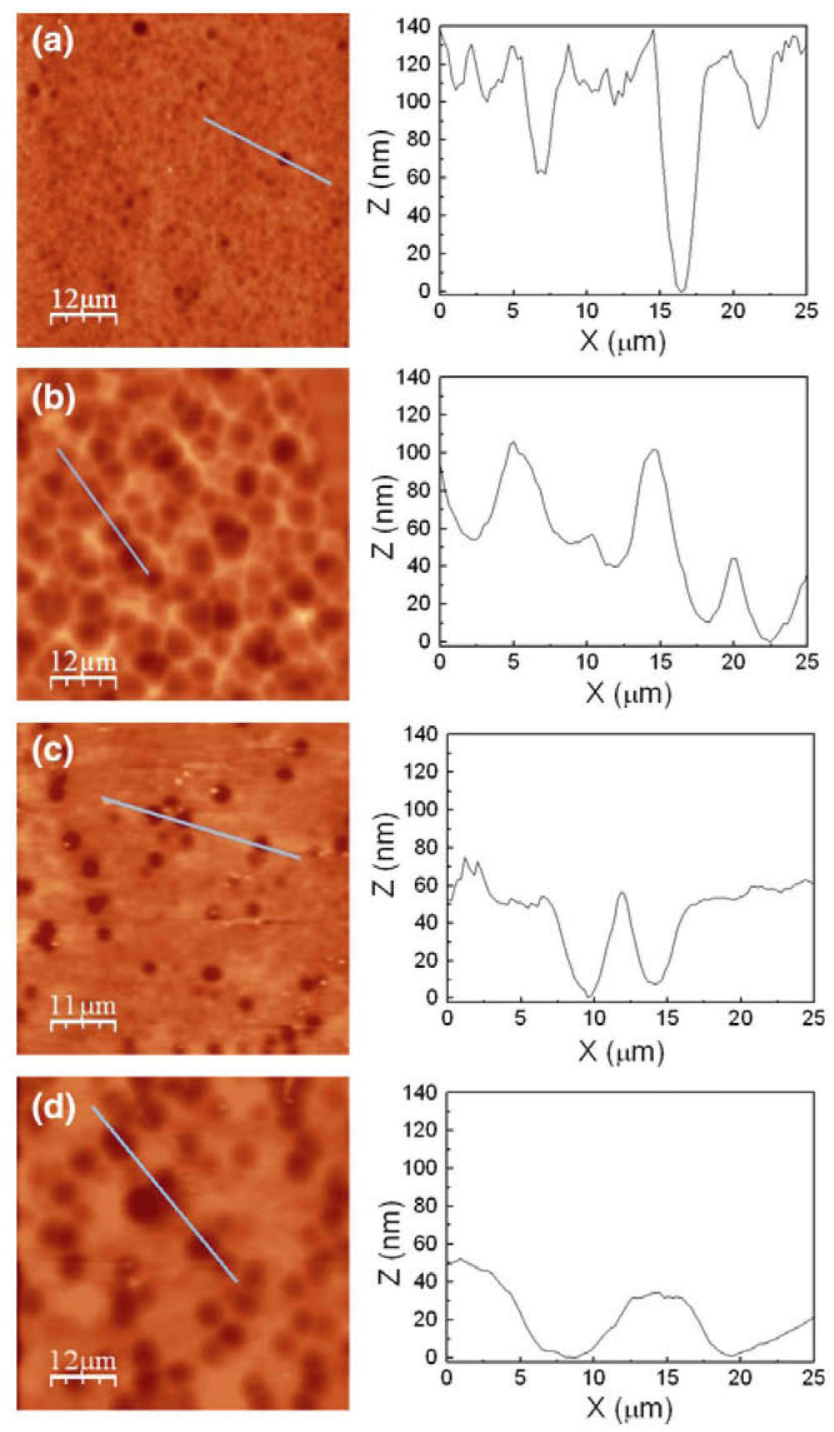

Fig. 4 AFM images and corresponding height profiles for (a and b) substrates etched and silica coatings annelaed at $60^{\circ} \mathrm{Cfor} 48 \mathrm{~h}$ on etched substrates (HF 40\%) for 20 (a and c) seconds and (b and d) $2 \mathrm{~min}$

not necessary to illuminate in a particular direction. Thus, we illuminate from the top of the sample to have a larger illuminated area and consequently to generate a larger number of photons. The distance traveled by light from the lighting source to the substrate (the edge of the glass in case of silica coating, and the glass surface in the case of quinine containing coating) is $1 \mathrm{~cm}$. Figure $7 \mathrm{c}$ presents a picture of the testing device. Figure $7 \mathrm{~d}$ shows a photo of a glass substrate partially coated inserted in the device and illuminated horizontally. We observe how the light is guided in the device escaping in the uncoated region.

Figure 8 present the light propagating decay measurements for silica coatings prepared over smooth substrates at different temperatures and for different number of layers.
We observed that the presence of the silica coating improves the light guiding inside the substrate. There is no clear dependence of the guided light with the annealing temperature. Only the $180^{\circ} \mathrm{C}$ treated coating exhibit larger light intensity clearly than the rest, probably related to a better densification that favors a smoother interface.

For a waveguide the light intensity decays as

$\mathrm{I}=\mathrm{I}_{\mathrm{o}} \mathrm{e}^{-\mathrm{x} / \mathrm{l}}$

being $\mathrm{x}$ the distance from the illumination point and 1 the decay length. None of the curves present this exponential decay. As the noise in the measurements is at maximum $10 \%$, we conclude that the decay in the measured range $(40 \mathrm{~mm})$ must be less than $10 \%$. According to Eq. 1 a decay of $10 \%$ in the light intensity correspond to $\mathrm{x} / \mathrm{l}=0.105$. As such a decay is not observed for a distance of $40 \mathrm{~mm}$, we may conclude that decay length of the waveguide, 1 , is over $400 \mathrm{~mm}$.

For the multilayers we find a tendency to increase the signal with the number of layers. This behavior is also related to a better densification (as we carry on an annealing process after each layer deposition).

In Fig. 8c, we observe that chemical etching of the substrate before being coated, does not affect significantly the light propagation. This is reasonable since the depth of the pores induced by the etching results significantly smaller than the wavelength of the light source.

The light guiding curves upon illumination with $365 \mathrm{~nm}$ light for the coating with quinine are presented in Fig. 9 where it can observe the optical behavior of the coating with different quinine concentrations (from 1 to $15 \%$ wt to Silica) (Fig. 9a), sintering temperatures (Fig. 9b), number of layers (Fig. 9c), and with different surface roughness (etching with HF) (Fig. 9d). The standard conditions are: monolayer coatings, sintering at $60^{\circ} \mathrm{C} / 48 \mathrm{~h}$ and not etching. In general, we observe these curves to exhibit a clear decrease with the distance, indicating that decay length is fairly smaller in these samples that in the pure silica coating. In the silica coating, due to the illumination geometry, only photons close to be parallel to the surface comes into the material (see Fig. 7a), so they are expected to suffer total reflection, propagating far away along the material. On the contrary, for the quinine containing coatings, the light is generated by photoluminescence and emitted in a more isotropic way, so there is a larger distribution of angles, and few photons will scatter the surface in total internal reflection. Hence, only a small fraction of the emitted photons will travel through the coatings in guiding conditions.

For quinine concentration up to $10 \%$, the light intensity is small and does not exhibit a clear distance dependence, while the coatings with quinine 12 and $15 \%$ the signal is fairly larger and shows a clear decay (Fig. 9a). 
Fig. 5 AFM images and for quinine doped (12\% wt) silica coatings deposited on soda lime substrates etched (HF $40 \%$ ) for (a) $20 \mathrm{~s} \mathrm{(b)} \mathrm{and} 2 \mathrm{~min}$ with their respective height profiles corresponding height profiles
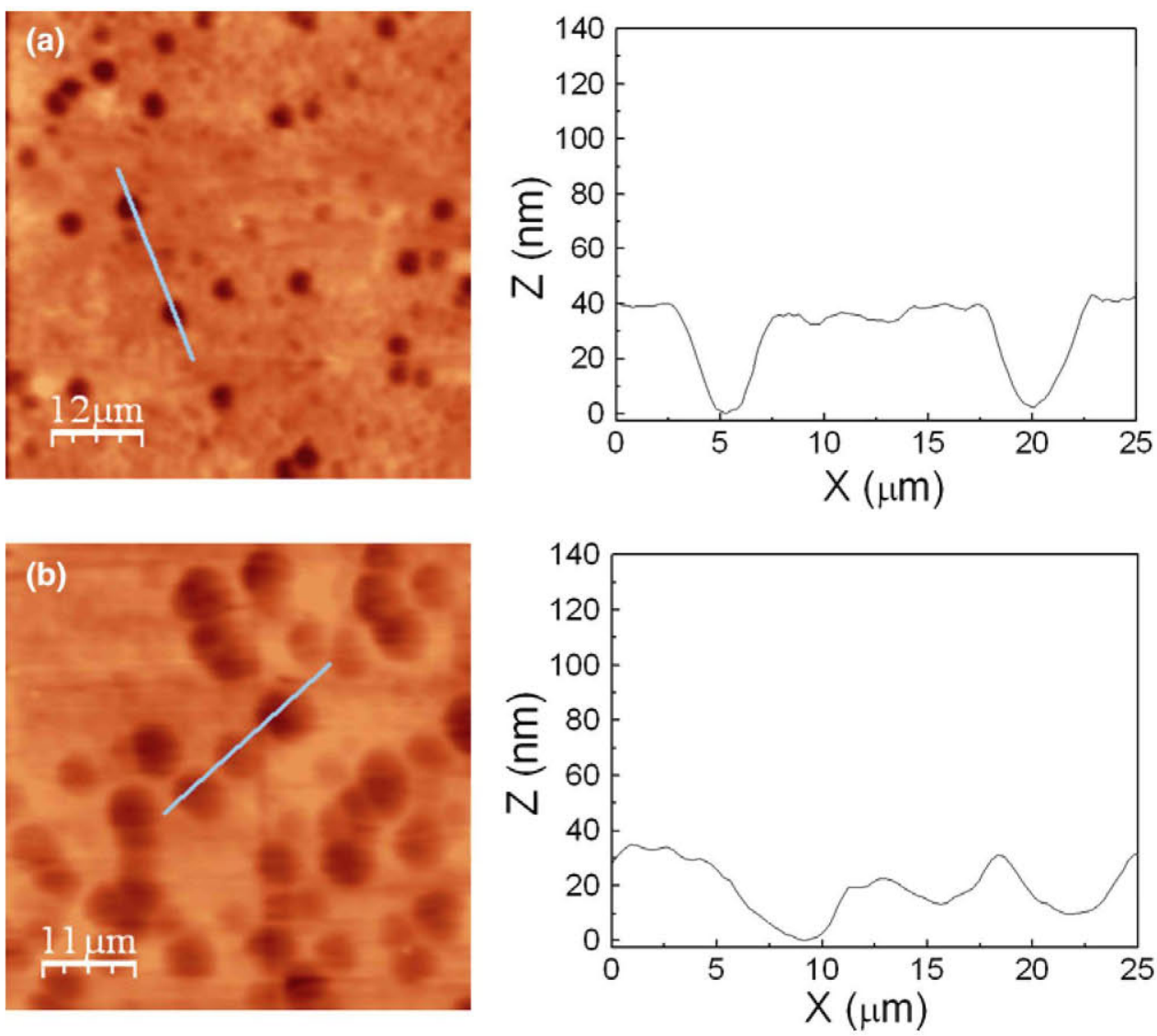

Fig. 6 Photoluminescence for quinine doped $(1-15 \%)$ silica coating consolidated at $60^{\circ} \mathrm{C}$ for $48 \mathrm{~h}$. a Emission spectrum upon excitation with $355 \mathrm{~nm}$ light and $\mathbf{b}$ excitation spectrum for the $433 \mathrm{~nm}$ emission (a)

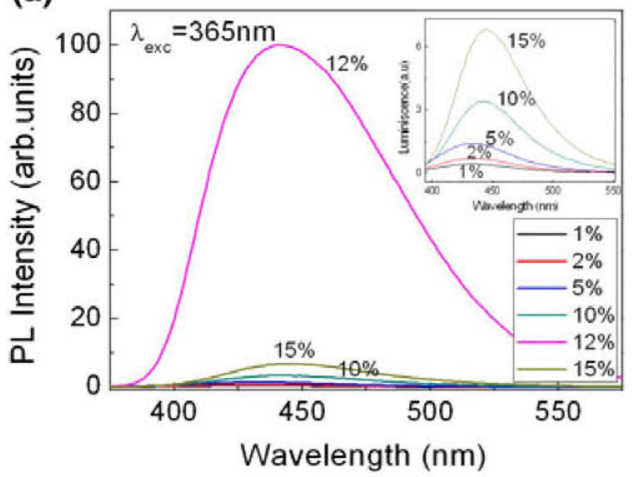

(b)

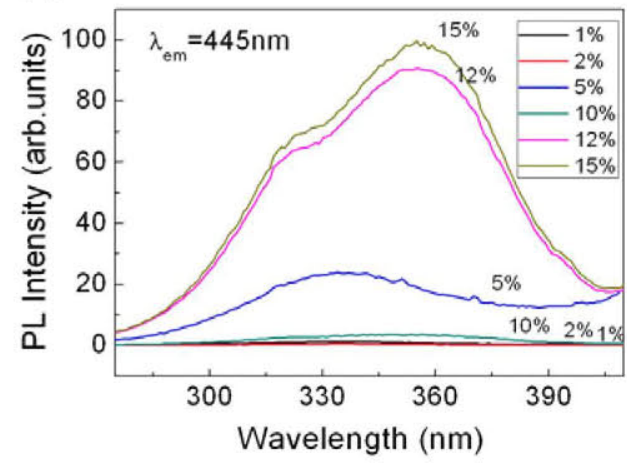

The curves corresponding to coatings with $12 \%$ quinine annealed at different temperatures are shown in Fig. 9b. The light intensity decreases with annealing temperature. Moreover, there is a meaningful reduction of intensity for temperatures above $60^{\circ} \mathrm{C}$, due to the thermal damage of the molecule. The light intensity increases with the coating thickness which is mainly related to the increase of quinine for unit area (Fig. 9c). All coatings exhibit a similar decay with distance, decreasing up to $50 \%$ over $4 \mathrm{~cm}$ as it can be seen in Table 1. Coatings deposited over etched exhibit a very weak photoluminescence intensity preventing us to observe clearly the decay with distance, as shown in Fig. 9d. This photoluminescence intensity reduction is likely related to the degradation of the quinine molecule in contact with rests of the etching solution (HF) on the substrates, as the quinine emission is quenched in presence of halides. From these measurements we estimate the decay length of the luminescence for the quinine containing 
Fig. 7 a, b Scheme of the devices developed to test wave guiding performance; $\mathbf{c}$ real picture of the device and d Photo of a pure silica coating inserted in the device and illuminated with blue light (Color figure online) (a)
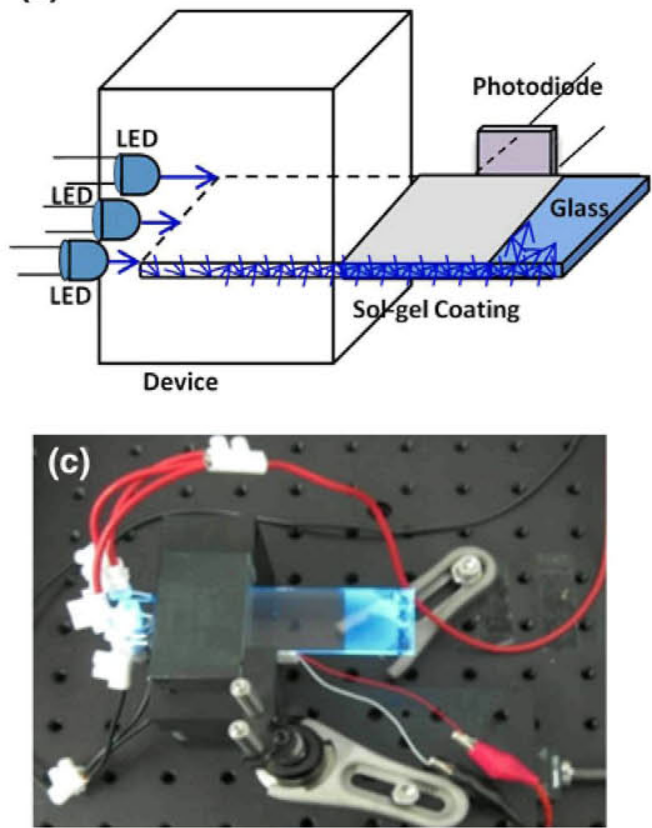

(b)

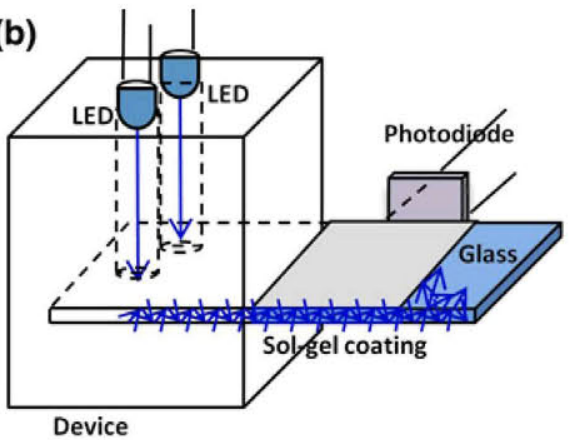

(d)

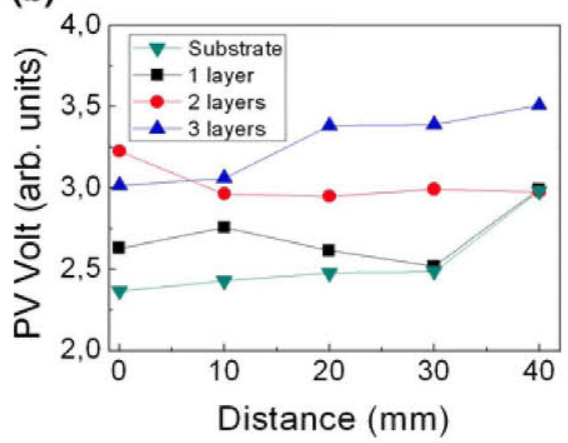

(c)

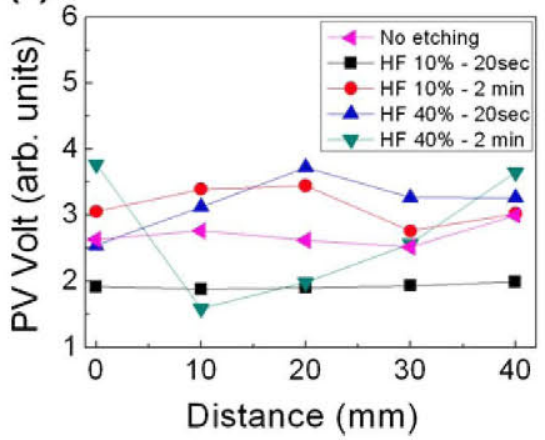

Fig. 8 Light propagation curves for silica coatings: a with different annealing conditions, b multilayers consolidated at $60{ }^{\circ} \mathrm{C}$ for $48 \mathrm{~h}$ with different number of layers and $\mathbf{c}$ for different etching of the substrate

coatings using Eq. 1. Results are presented in Table 1. This decay length of the order of few $\mathrm{cm}$, at least one order of magnitude below those of pure silica coatings.

For a light guiding device fabricated on a glass element it is required to promote both light guiding along the glass, and the expulsion at the desired region. It is possible to promote light escaping from glasses at desired regions by selective etching. However, the method is not valid in our case because etching promotes quinine degradation and, consequently, a significant reduction of photoluminescence intensity. In view of these results, the ideal device should consist on pure silica coating to guide the light and quinine doped regions at the areas to be luminous. UV light $(355 \mathrm{~nm})$ injected in the silica coated materials will travel with few looses over large distances. When this light reaches the regions with quinine it will excite quinine photoluminescence leading to blue light emission that leaves the substrate producing intense luminescence. Moreover, any loose in the silica coated region does not lead to observable light emission since it is UV light.

\section{Conclusions}

We successfully prepared pure and quinine doped silica coatings over sodalime glasses. The coatings can be consolidated preserving the quinine molecule that retains its fluorescent properties. We estimated the guiding light 
Fig. 9 Dependence of the light propagation in silica-quinine sol-gel coatings with a quinine concentration, $\mathbf{b}$ annealing temperature, $\mathbf{c}$ number of layers and $\mathbf{d}$ chemical etching of the substrate (a)

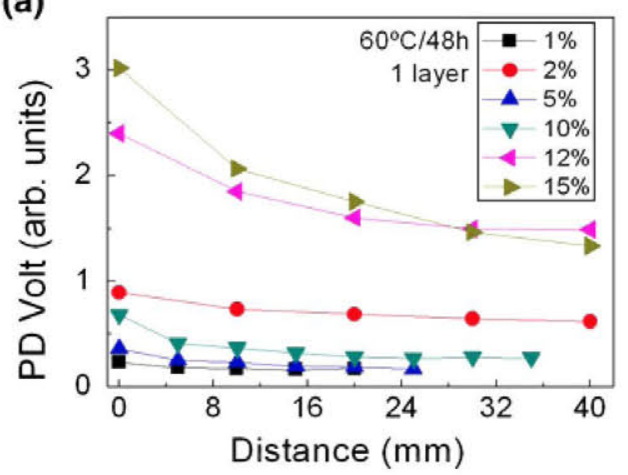

(c)

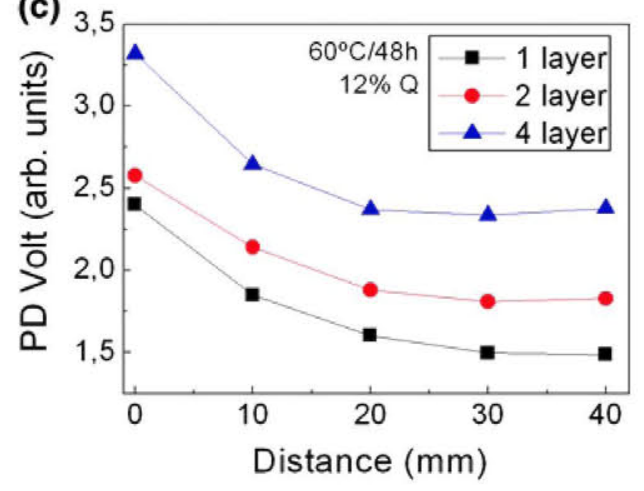

(b)

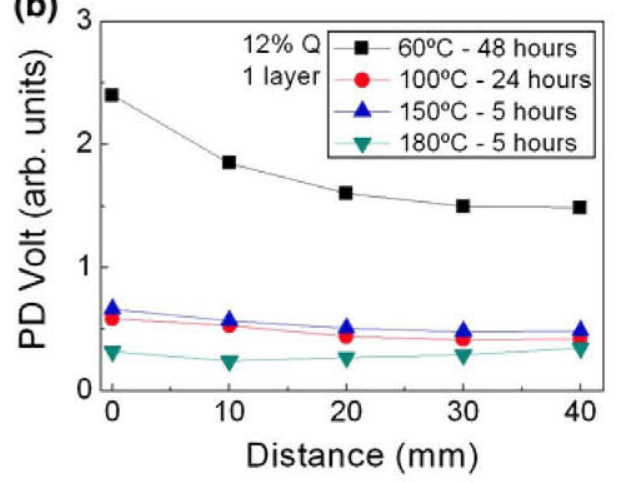

(d)

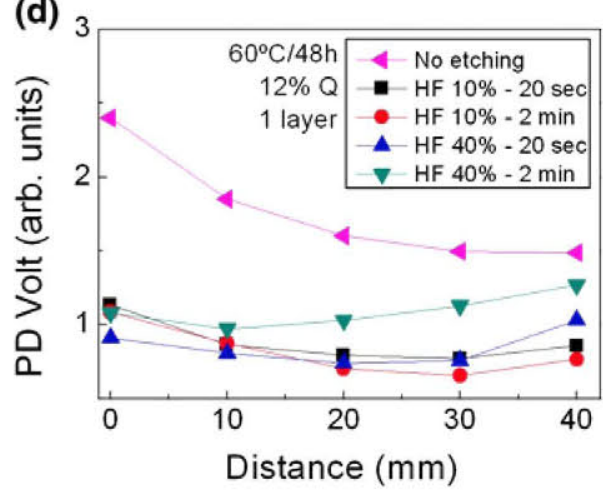

Table 1 Decay length of silica coatings with different concentrations of quinine consolidated at $60^{\circ} \mathrm{C}$ for $48 \mathrm{~h}$

\begin{tabular}{lc}
\hline $\begin{array}{l}\text { Quinine content } \\
(\% \mathrm{wt})\end{array}$ & $\begin{array}{l}\text { Decay length } \\
(\mathrm{cm})\end{array}$ \\
\hline 1 & 7.3 \\
2 & 11.6 \\
5 & 3.5 \\
10 & 4.4 \\
12 & 8.6 \\
15 & 5.1 \\
\hline
\end{tabular}

properties of the coating with a home-made device. The pure silica coatings allow to guide the light thought the sodalime glass over large distances (of the order of the meter) while quinine presence induces isotropic photoluminescence. Combination of both pure and quinine doped coating results interesting for the development of light guiding circuits and illuminate regions in glass elements without electronic circuits.

Acknowledgments This work was supported by the Spanish Ministry of Science and Innovation through the Projects MAT2010C21088-C03, FIS-2008-06249 and the Madrid Government project NANOBIOMAGNET S2009/MAT-1726.

\section{References}

1. Adams MJ (1981) An Introduction to optical waveguides. J. Willey, New York

2. Senior JM, Jamro MY (2009) Optical fiber communications: principles and practice. Pearsons Prentice Hall, USA

3. Townsend PD (1987) Rep Prog Phys 50:501

4. Mazzoldi P, Arnold GW, Battaglin G, Gonella F, Haglund RF Jr (1996) J Nonlinear Opt Phys Mater 5:285

5. Dubois JC, Gazard M, Ostrowsky DB (1973) Opt Commun 7:237

6. Choi C, Lin L, Liu Y, Choi J, Wang L, Haas D, Magera J, Chen RT (1998) J Lightwave Technol 22:2168

7. Gonella F, Caccavale F, Bogomolova LD, D'Acapito F, Quaranta A (1998) J Appl Phys 83:1200

8. Li A, Wang Z, Liu J, Zeng X (2011) Opt ND Lasers 49:351 in English

9. Hench LH, West JK (1990) Chem Rev 90:33

10. Brinker CJ, Scherer GW (1990) Sol-gel science: the physics and chemistry of sol-gel processing. Academic Press, San Diego

11. Klein LC (1988) Sol gel technology for thin films, fibers, performs electronics and specialty shapes. Noyes Publication, New Jersey

12. Sakka S (2004) Sol-gel science and technology. Processing, characterization, and applications. Kluwer Academic Press, Massachusetts

13. Righini GC, Pelli S (1997) J Sol Gel Sci Technol 8:991

14. Najafi SI, Touam T, Sara R, Andrews MP, Fardad MA (1998) J Lightwave Technol 16:1640

15. Yang L, Saavedra SS, Armstrong NR, Hayes J (1994) Anal Chem $66: 1254$ 
16. Villegas MA, García MA, Llopis J, Fernández-Navarro JM (1998) J Sol Gel Sci Technol 11:251

17. Arnold GW, De Marchi G, Gonella F, Mazzoldi P, Quaranta A, Battaglin G, Catalan M, Garrido F, Haglund RF Jr (1996) Nucl Instrum Methods Phys Res B 116:507

18. Montero EF, García MA, Villegas MA, Llopis J (2004) Bol Soc Esp Ceram V 43(1):8-11

19. Montero EF, García MA, Villegas MA, Llopis J (2008) Bol Soc Esp Ceram V 47(1):1-6

20. Shiu FM, Chen MH, Tang RF, Jeng YJ, Chang MY, Perng JH (1997) J Non-Cryst Solids 209:61

21. Meneses-Nava MA, Barbosa-García O, Díaz-Torres LA, ChávezCerda S, Torres-Cisneros M, King TA (2001) Opt Mater $17: 415-418$

22. Meneses-Nava MA, Barbosa-García O, Díaz-Torres LA, ChávezCerda S, Torres-Cisneros M, King TA (1999) Opt Mater 13:327-332

23. Spiers RP, Subbaraman CV, Wilkinson WL (1974) Chem Eng Sci 29:389

24. García MA, Paje SE, Llopis J, Villegas MA (2000) Bol Soc Esp.de Cerám. y Vidrio 39:641

25. Pakizeh M, Omidkhah MR, Zarringhalam A (2007) Int J Hydrog Energy 32:1825-1836
26. Asomoza M, Domínguez MP, Solís S, Lara VH, Bosch P, López T (1998) Mater Lett 36:249-253

27. Rey NA, Dos Santos KC, Menezes MÂBC, Mangrich AS, Pereira-Maia EC (2006) J Braz Chem Soc 17(3):497-504

28. Joshi GV, Pawar RR, Kevadiya BD, Bajaj HC (2011) Microporous Mesoporous Mater 142:542-548

29. García MA, García-Heras M, Con E, Bastidas JM, Villegas MA, Montero E, Llopis J, Sada C, De Marchi G, Battaglin G, Mazzoldi P (2004) J Appl Phys 96:3737

30. Wu LYL, Boon L, Chen Z, Zeng XT (2009) Thin Solid Films 517:4850-4856

31. Caruso R, Díaz-Parralejo A, Miranda P, Guiberteau F (2001) J Mater Res 16(8):2391-2398

32. Chen RF (1967) Anal Biochem 19:374

33. Schulman SG, Threatte RM, Capomacchia AC, Paul WL (1974) J Pharm Sci 63:876

34. Levy D, Avnirb D (1991) J Photochem Photobiol A Chem 57:41

35. Vitt JE, Engstrom RC (1997) Anal Chem 69:1070

36. Hosseini MS, Belador F (2009) J Hazard Mater 165:1062-1067

37. Lakowicz JR (2006) Principles of fluorescence spectroscopy. Springer, New York, pp 1-25

38. Mishra H, Pant D, Pant TC, Tripathi HB (2006) J Photochem Photobiol A Chem 177:197-204 